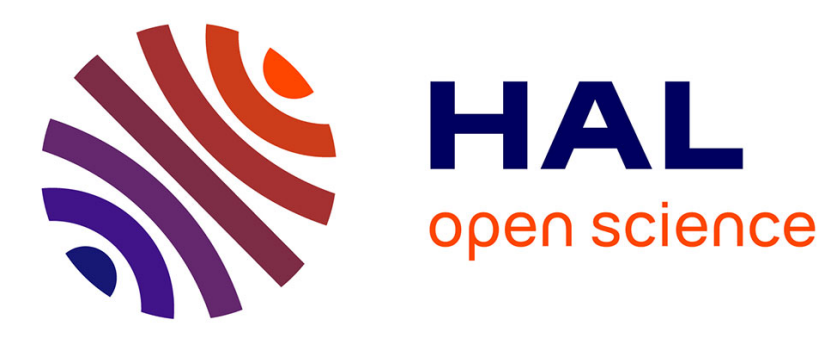

\title{
Pint: A Static Analyzer for Transient Dynamics of Qualitative Networks with IPython Interface

\author{
Loïc Paulevé
}

\section{To cite this version:}

Loïc Paulevé. Pint: A Static Analyzer for Transient Dynamics of Qualitative Networks with IPython Interface. CMSB 2017 - 15th conference on Computational Methods for Systems Biology, Sep 2017, Darmstadt, Germany. pp.370 - 316, 10.1007/978-3-319-67471-1_20 . hal-01589248

\section{HAL Id: hal-01589248 \\ https://hal.science/hal-01589248}

Submitted on 18 Sep 2017

HAL is a multi-disciplinary open access archive for the deposit and dissemination of scientific research documents, whether they are published or not. The documents may come from teaching and research institutions in France or abroad, or from public or private research centers.
L'archive ouverte pluridisciplinaire HAL, est destinée au dépôt et à la diffusion de documents scientifiques de niveau recherche, publiés ou non, émanant des établissements d'enseignement et de recherche français ou étrangers, des laboratoires publics ou privés. 


\title{
PINT: a Static Analyzer for Transient Dynamics of Qualitative Networks with IPython Interface
}

\author{
Loïc Paulevé \\ CNRS, LRI UMR 8623, Univ. Paris-Sud - CNRS \\ Université Paris-Saclay, 91405 Orsay, France \\ loic.pauleve@lri.fr
}

\begin{abstract}
The software PINT is devoted to the scalable analysis of the traces of automata networks, which encompass Boolean and discrete networks. PINT implements formal approximations of transient reachabilityrelated properties, including mutation prediction and model reduction. PINT is distributed with command line tools, as well as a Python module pypint. The latter provides a seamless integration with the Jupyter IPython notebook web interface, which allows to easily save, reuse, reproduce, and share workflows of model analysis.

PINT can address networks with hundreds to thousands interacting components, which are typically intractable with standard approaches.
\end{abstract}

\section{Introduction}

The computational analysis of the qualitative dynamics of biological networks faces the state space explosion problem, limiting the tractability of detailed models. Many studies have to use reduced models which often lose important properties and may lead to approximative results.

PINT provides formal and scalable analysis for the transient discrete dynamics (traces/trajectories) of automata networks, which subsume Boolean and multivalued networks. PINT implements an abstract interpretation of traces based on a static analysis of causality of transitions. It results in over- and underapproximations of PSPACE-complete problems by P. $\exp (k-1)$ and NP. $\exp (k-$ 1) problems, where $k$ is the number of qualitative levels of network nodes (2 for Boolean networks). PINT then relies on Boolean constraint satisfaction (SAT) and Answer-Set Programming (ASP, [3]) for their efficient resolution.

Besides simple transient reachability analysis (from state $s_{0}$ there exists a succession of transitions leading, even briefly, to a state satisfying a given property), PINT features include the prediction of mutations to control the reachability properties, the identification of bifurcation transitions responsible for differentiation processes, and model reduction which preserves transient reachability properties. For each case, returned results have formal guarantees on their correctness (under-approximations, satisfying sufficient conditions) or completeness (over-approximations, satisfying necessary conditions).

\footnotetext{
* This work was supported by ANR-FNR project "AlgoReCell" (ANR-16-CE12-0034) and by CNRS PEPS INS2I 2017 project "FoRCe".
} 
Most of PINT analysis can typically handle networks with several hundreds of components. PINT also provides interfaces with exact model-checkers, such as NuSMV [5], ITS [16] and Mole [25], taking advantage of implemented static model reduction to enhance their tractability on large models. Usual explicit reachable state graph analysis are also available, although other tools dedicated to Boolean or multi-valued networks already provide them, e.g., [10,14,18].

User Interfaces PINT can be invoked either using command line executables, suited for batch deployments, or through a programmable python interface. Moreover, its embedding in the Jupyter IPython notebook allows a user-friendly web interface to ease the management of models and calls to PINT. Jupyter notebooks provide a convenient environment for editing, saving, sharing, and reproducing model analysis workflows. It is a common framework for data-oriented bioinformatics tools $[2,6,12]$, and has promising suitability for computational systems biology, where reproducibility is very important as well.

Distribution PINT is written mainly with the OCaml programming language and is actively developed since 2011. It is distributed under the free software licence CeCILL, and is available at http://loicpauleve.name/pint where binary packages are provided for Ubuntu Linux and Mac OS X.

The Docker ${ }^{1}$ image pauleve/pint provides a ready-to-use PINT environment for usual operating systems (Windows, Mac OS X, Linux), and notably the Jupyter web interface. Such a kind of distribution becomes standard for providing accessible and reproducible analyses in bioinformatics, e.g., BioContainers [15].

\section{Input model}

PINT takes as input asynchronous automata networks specified in plain text. Automata networks are sets of finite-state machines having local transitions conditioned by the state of other automata in the network. The global state space of the network is the produce of the local states of individual automata, and transitions are applied non-deterministically.

Fig. 1 shows an example of automata networks with its plain text representation in PINT format. By convention, the file names end with . an.

Automata networks are expressive enough to encode the asynchronous semantics of Boolean and multi-valued networks. The main difference with these latter frameworks is the explicit specification of local transitions for each automaton (node) of the network, compared to a function-centred specification for Boolean and multi-valued networks $[7,19]$.

PINT can automatically convert models expressed as Boolean or multi-valued networks using the pint-import command or pypint.load() python function. Most of the conversions are performed using GINsIM [10], enabling the support for SBML-qual, GINsim, as well as various text formats. Models can be directly imported from URLs and from CellCollective database [13]. BIOCHAM reaction networks are also supported, following their Boolean semantics [4].

\footnotetext{
1 http://docker.com
} 


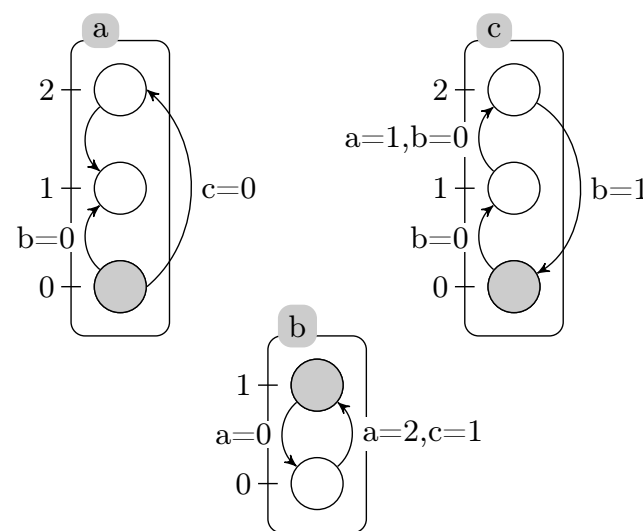

(a) Automata network a $[0,1,2]$

b $[0,1]$

c $[0,1,2]$
$0 \rightarrow 1$ when $b=0$

a $0 \rightarrow 2$ when $c=0$

a $2 \rightarrow 1$

b $1 \rightarrow 0$ when $a=0$

b $0 \rightarrow 1$ when $a=2$ and $c=1$

c $0 \rightarrow 1$ when $b=0$

c $1 \rightarrow 2$ when $a=1$ and $b=0$

c $2 \rightarrow 0$ when $b=1$

initial_state $a=0, b=1, c=0$

(b) PINT textual format

Fig. 1: (a) graphical representation of an automata network: automata are labelled boxes and their local states by circles where ticks are their identifier within the automaton. The initial state is composed of the local states in gray. A local transition is a directed edge between two local states of an automaton. Transitions can be labelled with states of other automata which are necessary to trigger the transition. (b) equivalent PINT plain text representation

\section{$3 \quad$ Main features and benchmarks}

The main originality of PINT resides in the static analysis for transient reachability properties: such an approach avoids building the reachable state transition graph, neither explicitly nor symbolically. Therefore, the analysis aims at being tractable on large networks, at the price of giving possibly incomplete results.

We present the related features, illustrated in Fig. 2, with benchmarks to support their tractability on large biological networks. Computation times have been obtained on an Intel(R Core ${ }^{\mathrm{TM}} \mathrm{i} 7-47703.40 \mathrm{GHz}$ CPU with 16GiB RAM.

Reachability analysis: formal approximation and model reduction Given an initial state, a usual problem is to determine the existence of a sequence of transitions which leads to the activation or de-activation of key components (e.g., transcription factors) or to a particular attractor. Reachability verification is a PSPACE-complete problem and its resolution often explodes on large networks. PINT implements over- and under-approximation of reachability $[21,9]$ which allow tackling large models, although being potentially inconclusive when the over-approximation is satisfied but not the under-approximation. In such cases, one should fall back to classical model-checking. To that aim, the goal-oriented reduction [19] identifies transitions that do not contribute to the goal reachability, and hence can be removed prior to the reachability analysis. This model reduction preserves all minimal traces to the goal, and can enhance greatly the tractability of model-checking. See Table 1 and [19] for benchmarks. 


\begin{tabular}{|c|c|c|c|c|c|}
\hline & \multirow{4}{*}{$\begin{array}{r}|\mathrm{T}| \\
381 \\
\mathbf{0}\end{array}$} & \multirow{4}{*}{$\begin{array}{r}\mid \text { states } \mid \\
\approx 2.4 \cdot 10^{8} \\
\mathbf{1}\end{array}$} & \multicolumn{3}{|c|}{ Verification of goal reachability } \\
\hline \multirow{3}{*}{$\begin{array}{l}\text { Model }(\mid \text { nodes } \mid) \\
\text { TCell-d (101) [1] } \\
\text { profile } 1\end{array}$} & & & NuSMV (EF $g$ ) & ITS-REACH & \multirow{3}{*}{$\frac{\text { PINT }}{0.02 \mathrm{~s}}$} \\
\hline & & & $2 \mathrm{~s} \quad 40 \mathrm{Mb}$ & $0.5 \mathrm{~s} \quad 26 \mathrm{Mb}$ & \\
\hline & & & & & \\
\hline \multirow{2}{*}{$\begin{array}{l}\text { TCell-d (101) } \\
\text { profile } 2\end{array}$} & 381 & $\mathrm{KO}$ & $\mathrm{KO}$ & $1.6 \mathrm{~Gb}$ & \multirow[t]{2}{*}{$4.5 \mathrm{~s}$} \\
\hline & 221 & $75,947,684$ & $470 \mathrm{~s} \quad 270 \mathrm{Mb}$ & $160 \mathrm{Mb}$ & \\
\hline \multirow{2}{*}{ RBE2F (370) [22] } & 742 & $\mathrm{KO}$ & $\mathrm{KO}$ & $\mathrm{KO}$ & \multirow[t]{2}{*}{$0.2 \mathrm{~s}$} \\
\hline & 56 & $2,350,494$ & $3 \mathrm{~s} \quad 37 \mathrm{Mb}$ & $4 \mathrm{~s} \quad 13 \mathrm{Mb}$ & \\
\hline \multirow{2}{*}{ MAPK (309) [24] } & 1251 & $\mathrm{KO}$ & $\mathrm{KO}$ & $\mathrm{KO}$ & \multirow[t]{2}{*}{$48 \mathrm{~s}$} \\
\hline & 429 & KO & KO & KO & \\
\hline
\end{tabular}

Table 1: Benchmark ${ }^{\dagger}$ of goal reachability verification with two exact methods (NuSMV and ITS-REACH) and PINT, before (normal font) and after (bold font) goal-oriented model reduction; $|\mathrm{T}|$ is the number of local transitions in automata networks; |state| is the number of reachable global states, when computable. KO indicates an out-of-memory/time computation. In all cases PINT is conclusive.

\begin{tabular}{|c|c|c|c|c|c|c|c|c|}
\hline Goal & \multicolumn{2}{|c|}{$\begin{array}{c}\text { TCell-d (101) } \\
\text { FOXP3 }=1\end{array}$} & \multicolumn{2}{|c|}{$\begin{array}{l}\text { Egf-r (104) [23] } \\
\text { AP1 }=1\end{array}$} & \multicolumn{2}{|c|}{$\begin{array}{l}\text { MAPK }(309) \\
\text { ERK-PP=1 }\end{array}$} & \multicolumn{2}{|c|}{$\begin{array}{c}\text { PID }(10,229)[20] \\
\text { SNAIL }=1\end{array}$} \\
\hline 3-cut sets & $0.06 \mathrm{~s}$ & 35 & $0.02 \mathrm{~s}$ & 34 & $0.06 \mathrm{~s}$ & 24 & $1.2 \mathrm{~s}$ & 7 \\
\hline 4-cut sets & $0.10 \mathrm{~s}$ & 101 & $0.02 \mathrm{~s}$ & 34 & $0.1 \mathrm{~s}$ & 48 & $5 \mathrm{~s}$ & 37 \\
\hline 6-cut sets & $0.60 \mathrm{~s}$ & 495 & $0.03 \mathrm{~s}$ & 34 & $1 \mathrm{~s}$ & 60 & $10 \mathrm{~m}$ & 907 \\
\hline 3-mutations & $0.30 \mathrm{~s}$ & 15 & $0.30 \mathrm{~s}$ & 20 & $5 \mathrm{~s}$ & 222 & $50 \mathrm{~m}$ & 7 \\
\hline 4-mutations & $0.30 \mathrm{~s}$ & 15 & $0.30 \mathrm{~s}$ & 22 & $10 \mathrm{~s}$ & 1896 & $50 \mathrm{~m}$ & 67 \\
\hline 6-mutations & $0.30 \mathrm{~s}$ & 15 & $0.30 \mathrm{~s}$ & 22 & & & $50 \mathrm{~m}$ & 367 \\
\hline
\end{tabular}

Table 2: Performance ${ }^{\dagger}$ of cut sets and mutations under-approximations with PINT depending on the maximal cardinality of returned sets.

\begin{tabular}{|l|r|r|l||c|c||c|c|}
\hline & $|\mathrm{T}|$ & \multirow{2}{*}{$\mid$ states $\mid$} & \multicolumn{2}{|c||}{ goal } & \multicolumn{2}{|c||}{ NuSMV } & \multicolumn{2}{|c|}{ PINT } \\
\cline { 5 - 8 } & & & & $\left|t_{b}\right|$ & time & $\left|t_{b}\right|$ & time \\
\hline EGF/TNF (28) [17] & 53 & 3968 & NFkB $=0$ & 5 & $0.2 \mathrm{~s}$ & 2 & $0.1 \mathrm{~s}$ \\
MAPK (53) [11] & 173 & KO & Proliferation =1 & \multicolumn{2}{|c}{ KO } & 13 & $40 \mathrm{~s}$ \\
TCell-d (101) & 381 & KO & FOXP3 $=1$ & \multicolumn{2}{|c|}{ KO } & 4 & $58 \mathrm{~s}$ \\
\hline
\end{tabular}

Table 3: Performance ${ }^{\dagger}$ of exact and approximated identification of bifurcation transitions with NUSMV and PINT, respectively; $\left|t_{b}\right|$ is the number of identified bifurcation transitions.

${ }^{\dagger}$ Scripts and models available at http://loicpauleve.name/pint-benchmarks.tbz2 

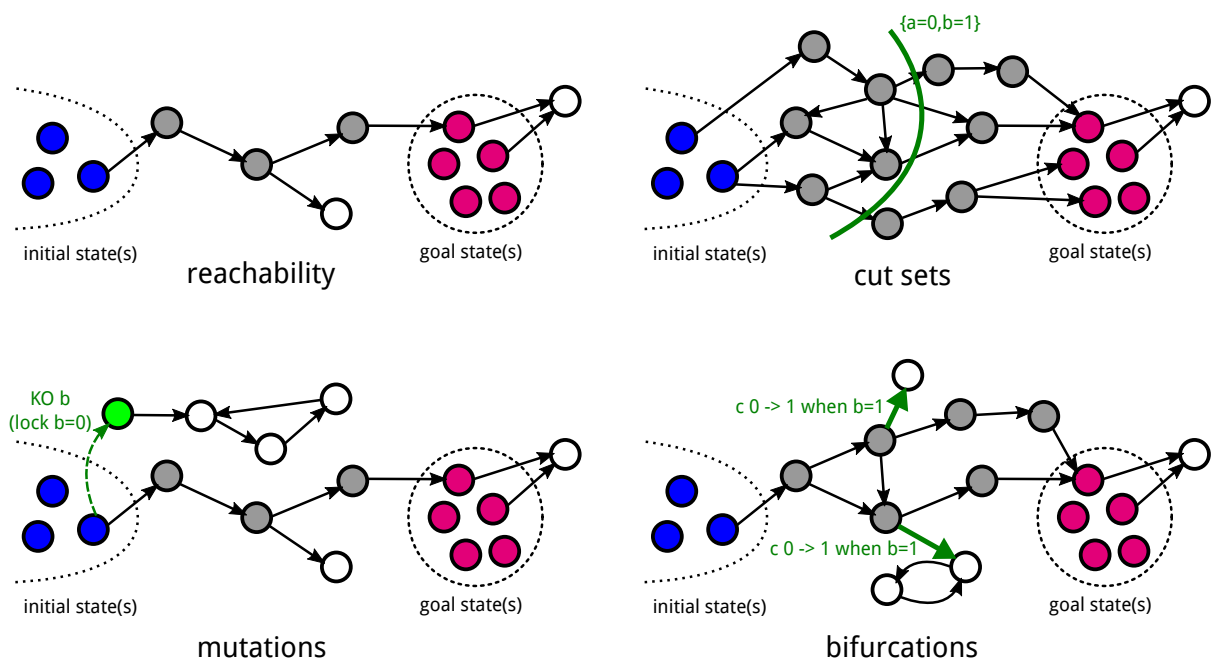

Fig. 2: Illustration of main features of PINT related to the transient reachability of a set of goal states from (a set of) initial state(s). Circles represent global states of the network and plain arrows dynamical transitions. Gray (resp. white) states are states which are (resp. are not) connected to a goal state.

Prediction of mutations for controlling reachability - Given an initial state and a goal state of interest, PINT provides several methods to control the transient reachability of the goal.

The most scalable approach identifies cut sets of all the paths of transitions leading to the goal. A cut sets consists in one or several local states of automata which are necessary for the goal reachability: if one prevents the transitions involving these local states, the goal is disconnected from the initial state. PINT provides extremely scalable under-approximation of cut sets [20], which is tractable on Boolean networks with thousands of nodes (Table 2). Cut sets can thus be implemented as mutations which lock automata to its initial local state.

An alternative approach relies on a combination of static analysis and SAT solving and allows to directly infer mutations (gain or loss of function) which prevent the goal reachability. Whereas less scalable than cut set computations, it provides in general complementary solutions to cut sets, notably by identifying mutations which modify the initial state of the network.

Identification of bifurcation transitions - PINT implements static analysis for identifying so-called bifurcation transitions [8] after which the systems loses the capability to reach a given goal. Bifurcation transitions correspond to local transitions of the automata network which turn out to be important decision steps during differentiation processes. They can be fully identified by modelchecking, but the static analysis in PINT allows tackling larger models, at the price of returning incomplete results (Table 3). 


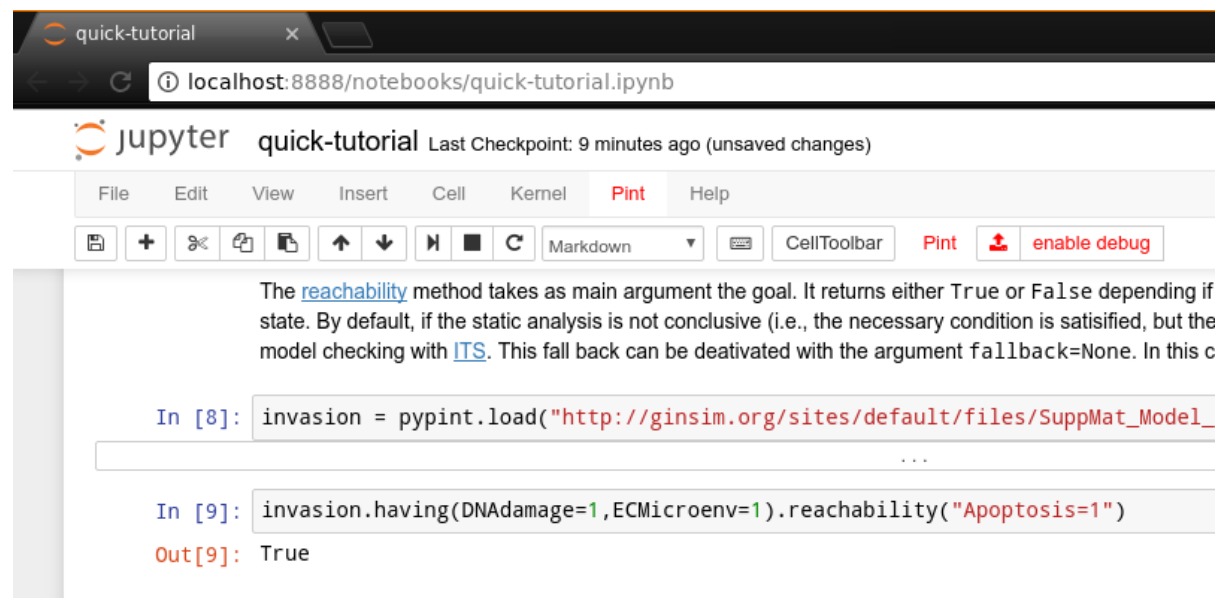

Fig. 3: Screen capture of Jupyter web interface running pypint in a notebook.

\section{Integration with Jupyter IPython Web Notebook}

Jupyter (http://jupyter.org) provides an interactive web interface for creating documents, named notebooks, which contain code, equations, and formatted texts. A notebook typically describes a full workflow of analysis, both with textual explanations and the full code and parameters to reproduce the results. It is a very popular framework in data science, including in bioinformatics $[6,12]$. A notebook is a single file which can be easily modified, shared, re-executed, and visualized online. For instance, the companion quick tutorial is available at http://nbviewer.jupyter.org/github/pauleve/pint/blob/ master/notebook/quick-tutorial.ipynb.

The pypint module provides custom integration within the Jupyter IPython notebook, with custom menus and actions for loading models and executing PINT commands, as well as direct visualization of data structures. See Fig. 3 and the companion quick tutorial for a preview.

\section{Conclusion}

In this paper, we presented the prominent features of PINT on the static analysis for transient reachability of automata networks, from property verification to inference, which are tractable on large biological networks. PINT also implements classical state transition graph analysis, from fixpoint computation (using SAT solving) to explicit state space exploration, with a limited scalability. A tour of features is given at https://loicpauleve.name/pint/doc/\#Tutorial.

In the next major release, we plan to add full support for synchronized local transitions, i.e., transitions that modify simultaneously the state of several automata. This improvement will allow to import any safe (1-bounded) Petri nets, broadening the class of supported dynamical models. 


\section{References}

1. W. Abou-Jaoudé, P. T. Monteiro, A. Naldi, M. Grandclaudon, V. Soumelis, C. Chaouiya, and D. Thieffry. Model checking to assess t-helper cell plasticity. Frontiers in Bioengineering and Biotechnology, 2, Jan 2015.

2. T. Antao. Bioinformatics with Python cookbook. Packt Publishing Ltd, 2015.

3. C. Baral. Knowledge Representation, Reasoning and Declarative Problem Solving. Cambridge University Press, New York, NY, USA, 2003.

4. L. Calzone, F. Fages, and S. Soliman. Biocham: an environment for modeling biological systems and formalizing experimental knowledge. Bioinformatics, 22(14):1805-1807, 2006.

5. A. Cimatti, E. Clarke, E. Giunchiglia, F. Giunchiglia, M. Pistore, M. Roveri, R. Sebastiani, and A. Tacchella. NuSMV 2: An opensource tool for symbolic model checking. In Computer Aided Verification, volume 2404 of Lecture Notes in Computer Science, pages 241-268. Springer Berlin / Heidelberg, 2002.

6. P. J. A. Cock, T. Antao, J. T. Chang, B. A. Chapman, C. J. Cox, A. Dalke, I. Friedberg, T. Hamelryck, F. Kauff, B. Wilczynski, and M. J. L. de Hoon. Biopython: freely available python tools for computational molecular biology and bioinformatics. Bioinformatics, 25(11):1422-1423, mar 2009.

7. F. Fages, T. Martinez, D. A. Rosenblueth, and S. Soliman. Influence Systems vs Reaction Systems, pages 98-115. Springer International Publishing, Cham, 2016.

8. L. F. Fitime, O. Roux, C. Guziolowski, and L. Paulevé. Identification of bifurcations in biological regulatory networks using answer-set programming. In Constraint-Based Methods for Bioinformatics Workshop, 2016.

9. M. Folschette, L. Paulevé, M. Magnin, and O. Roux. Sufficient conditions for reachability in automata networks with priorities. Theoretical Computer Science, 608, Part 1, From Computer Science to Biology and Back:66 - 83, 2015.

10. A. G. Gonzalez, A. Naldi, L. Sánchez, D. Thieffry, and C. Chaouiya. Ginsim: A software suite for the qualitative modelling, simulation and analysis of regulatory networks. Biosystems, 84(2):91 - 100, 2006. Dynamical Modeling of Biological Regulatory Networks.

11. L. Grieco, L. Calzone, I. Bernard-Pierrot, F. Radvanyi, B. Kahn-Perlès, and D. Thieffry. Integrative modelling of the influence of MAPK network on cancer cell fate decision. PLoS Comput Biol, 9(10):e1003286, oct 2013.

12. R. Grunberg, M. Nilges, and J. Leckner. Biskit - a software platform for structural bioinformatics. Bioinformatics, 23(6):769-770, jan 2007.

13. T. Helikar, B. Kowal, S. McClenathan, M. Bruckner, T. Rowley, A. Madrahimov, B. Wicks, M. Shrestha, K. Limbu, and J. A. Rogers. The cell collective: Toward an open and collaborative approach to systems biology. BMC Systems Biology, 6(1):96, 2012.

14. H. Klarner, A. Streck, and H. Siebert. PyBoolNet: a python package for the generation, analysis and visualization of boolean networks. Bioinformatics, page btw682, oct 2016.

15. F. d. V. Leprevost, B. A. Grüning, S. Alves Aflitos, H. L. Röst, J. Uszkoreit, H. Barsnes, M. Vaudel, P. Moreno, L. Gatto, J. Weber, M. Bai, R. C. Jimenez, T. Sachsenberg, J. Pfeuffer, R. Vera Alvarez, J. Griss, A. I. Nesvizhskii, and Y. Perez-Riverol. Biocontainers: An open-source and community-driven framework for software standardization. Bioinformatics (Oxford, England), Mar. 2017.

16. LIP6/Move. Its tools. http://ddd.lip6.fr/itstools.php. 
17. A. MacNamara, C. Terfve, D. Henriques, B. P. Bernabé, and J. Saez-Rodriguez. State-time spectrum of signal transduction logic models. Physical Biology, 9(4):045003, 2012.

18. C. Mussel, M. Hopfensitz, and H. A. Kestler. BoolNet - an R package for generation, reconstruction and analysis of boolean networks. Bioinformatics, 26(10):1378-1380, 2010.

19. L. Paulevé. Goal-Oriented Reduction of Automata Networks. In CMSB 2016 14th conference on Computational Methods for Systems Biology, volume 9859 of Lecture Notes in Bioinformatics. Springer, 2016.

20. L. Paulevé, G. Andrieux, and H. Koeppl. Under-approximating cut sets for reachability in large scale automata networks. In N. Sharygina and H. Veith, editors, Computer Aided Verification, volume 8044 of Lecture Notes in Computer Science, pages 69-84. Springer Berlin Heidelberg, Berlin, Heidelberg, 2013.

21. L. Paulevé, M. Magnin, and O. Roux. Static analysis of biological regulatory networks dynamics using abstract interpretation. Mathematical Structures in Computer Science, 22(04):651-685, 2012.

22. A. Rougny, C. Froidevaux, L. Calzone, and L. Paulevé. Qualitative dynamics semantics for SBGN process description. BMC Systems Biology, 10(1):1-24, 2016.

23. R. Samaga, J. Saez-Rodriguez, L. G. Alexopoulos, P. K. Sorger, and S. Klamt. The logic of egfr/erbb signaling: Theoretical properties and analysis of high-throughput data. PLoS Comput Biol, 5(8):e1000438, 082009.

24. B. Schoeberl, C. Eichler-Jonsson, E. D. Gilles, and G. Müller. Computational modeling of the dynamics of the map kinase cascade activated by surface and internalized egf receptors. Nature biotechnology, 20(4):370-375, 2002.

25. S. Schwoon. Mole. http://www.lsv.ens-cachan.fr/ schwoon/tools/mole/. 